\title{
Development of amperometric gas sensors for industrial/environmental usage
}

\author{
Hiroshi IMAYA ${ }^{1}$, \\ ${ }^{1}$ Riken Keiki Co., Ltd., 2-3, Minamisakae-cho, Kasukabe, Saitama, Japan,
}

\begin{abstract}
In 1974, toxic gas sensor by using a gas permeable electrode was first reported for CO monitoring. A lot of electrochemical gas sensors became available for detection of many different toxic gases in ppm ranges, by using the gas permeable electrode. The sensor has advantage in term of sensitivity, selectivity and power consumption which is suitable in practical fields. We have continued to develop more reliable electrochemical sensors by improving electrode materials, structure of electrode surface and electrolytic solutions. This paper describes our improvement techniques for electrochemical gas sensors.
\end{abstract}

Key words: Amperometric gas sensor, Electrochemical gas sensor, toxic gas detection, Electrode, gas permeable membrane

\section{Introduction}

In 1970s, gas permeable membrane electrode, which is porous polymer membrane coated on Pt particle, was developed by NASA through the fuel cell development for the space usage. In 1974, Amperometric sensor for CO gas detection was reported by using the gas permeable membrane electrode by Blurton et al [1].

Amperometric gas sensors have been widely used for industrial purposes and environmental monitoring. The electrochemical gas sensor has a very simple structure and works similar to a fuel cell. The sensor is constructed by a gas permeable membrane coated by a thin metal electrode. A current with electrochemical reaction of gases proceeding at the electrode/electrolyte solution/gas interface is detected as the gas sensing signal.

\section{Amperometric gas sensor}

\section{【Structure】}

A porous membrane shown in Fig. 1, coated on the inner surface with noble metal such as gold or platinum as an electrode is installed at the face of a plastic container filled with electrolyte (Fig. 1).

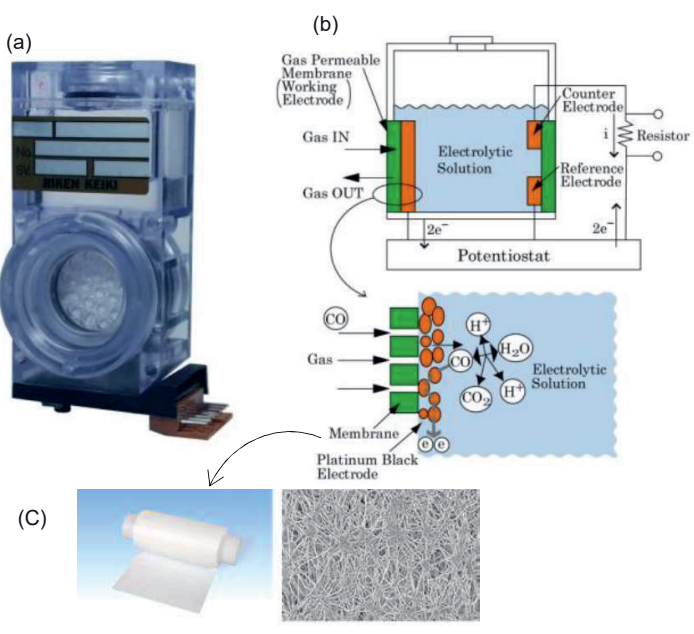

Fig. 1 Appearance of sensor(a), schematic diagram of an amperometric gas sensor for the detection of $\mathrm{CO}$ gas(b) and gas permeable membrane(c).

\section{【Principle of Detection】}

A working electrode and a reference electrode are held at a fixed potential by a potentiostat circuit. The gas to be detected is directly electrolyzed as the working electrode. For example, in case of Carbon monoxide(CO), the following chemical reaction occurs: 
Working electrode :

$$
\mathrm{CO}+\mathrm{H}_{2} \mathrm{O} \rightarrow \mathrm{CO}_{2}+2 \mathrm{H}^{+}+2 \mathrm{e}^{-}
$$

Counter electrode :

$$
\mathrm{O}_{2}+6 \mathrm{H}^{+}+6 \mathrm{e}^{-} \rightarrow 3 \mathrm{H}_{2} \mathrm{O}
$$

Current which is generated by the chemical reaction above is in proportion to gas concentration. On the equation $1, F, A, D, n$ and $t$ are constant. Therefore, gas concentration can be determined as shown equation 1 .

$I=(F \times A \times D \times c \times n) / t$

I:Detection current

F: Faraday constant

A: Electrode surface area

D: Gas diffusion coefficient

C: Gas concentration(ppm)

n: Electron number

t: time(s)

We are investigating and develping many amperometric gas sensors to enhance these sensor characteristics such as sensitivity and selectivity for the practical usage.

In this presentation, we introduce the some topics from our invetigations.

\section{Results and disccussion}

To enhance a sensor characteristics, studies have been made to improve the electrode, gas permeable membrane, elctrolytes for sensors.

\section{Modification of gas permeable membrane electrode}

As a new approach of improvement of the sensor characteristics, we applied ion implantation technology to modify the gas permeable membrane

polytetrafluoroethylene (PTFE).

The ion implantation into PTFE is expected to change the gas permeability of the membrane and nature of the metal electrode coated on the modified membrane. I will present effects of ion implantation into the gas permeable membrane on the sensitivity and selectivity of gases, such as $\mathrm{CO}, \mathrm{H}_{2}$, and other toxic gases.

Ion implantations of several kinds of ion into PTFE membranes with fluenses of $1 \times 10^{14} \sim 5 \times$ $10^{15}$ ions $/ \mathrm{cm}^{2}$ were carried out to modify the gas permeable membranes. Figure 2 shows a typical result of relationship between output current of the sensors and fluense of ions for $\mathrm{CO}$ gas. Sensors constructed with the ionimplanted gas permeable electrode showed 5.5 $\sim 38$ times higher sensitivity than that used non-ion- implanted one.
The morphology change is an important effect induced by the ion beam radiation, which brings characteristic change of the interfacial reaction of gases. The enhancement of sensitivity with the ion implantation is due to change of active nature of the Au electrode formed on the modified membrane and to increase of the effective surface area of the $\mathrm{Au}$ electrode.

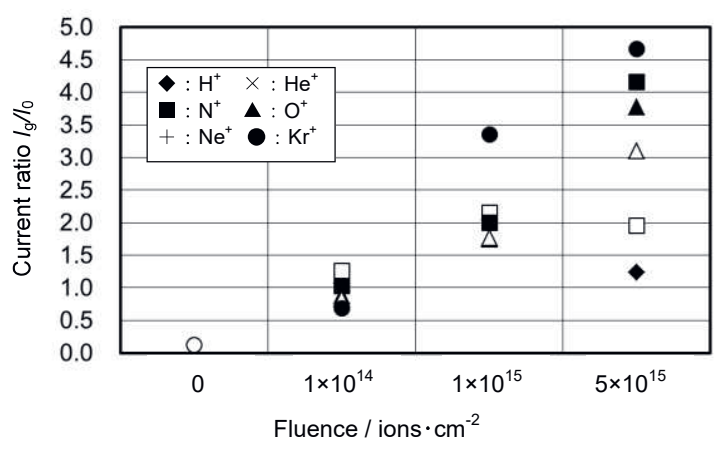

Fig. 2 Relationship between output current and fluence of the ion implantations with various kinds of ions, for 1000 ppm CO gas.

\section{Selective $\mathrm{HF} / \mathrm{HCl}$ gas sensor}

Amperometric sensors for the detection of acidic gases were investigated. A detection method is based on the electrode reactions of halogen liberated by the hydrogen ion in the electrolytic solutions containing various halide/halate ions. We have compared the detection properties of the sensors using different combinations of the halide/halate to detect different acidic gases, such as HF and $\mathrm{HCl}$.

We found that the sensitivity of the sensors depend on the solution composition and the acidic gases[2]. To detect $\mathrm{HF}$ gas, the $\mathrm{I}^{-} / \mathrm{IO}_{3}{ }^{-}$ system shows the highest sensitivity, however, the $\mathrm{Br}^{-} / \mathrm{BrO}_{3}{ }^{-}$gives hardly response. In the case of $\mathrm{HCl}$ gas, $\mathrm{I}^{-} / \mathrm{IO}_{3}{ }^{-}$system gives high sensitivity, but the $\mathrm{Br}^{-} / \mathrm{BrO}_{3}{ }^{-}$also adaptable to the sensing. From such different response to the different acidic gases, the choice or the combination of the halide/harate systems is effective to purposes of selective sensing or detection of the hole amount of the acidic gases.

\section{References}

[1] K. F. Blurton and J . M. Sedlak, J. Electrochem. Soc., 121(10),1315-1317(1974)

[2] H. Imaya, T. Ishiji and K. Takahashi, Sensors and Actuators B: Chemical, 108, Issues 1-2, 803807(2005) 